\title{
Original
}

\section{Matrix Metalloproteinases in Cervical Intervertebral Discs : A Comparison of Image-Indicated Disease Symptoms and the Manifestation of Matrix Metalloproteinases}

\author{
Yushi Hoshino ${ }^{1)}$, Yutaka Hiraizumi ${ }^{1)}$, Hideyo MiYaOKa ${ }^{1)}$, \\ Kayoko ToNo $^{2)}$ and Tetsuhiko $\mathrm{TACHIKAWA}^{2)}$
}

\begin{abstract}
We report the study of matrix metalloproteinases (MMPs) in cervical intervertebral discs, immunohistochemically, and by comparing the manifestation of MMPs with X ray-based side views and MRI visual imaging. We analyzed intervetebral disc tissue samples from 20 patients who had undergone surgery for herniation of their cervical intervertebral discs and cervical spondylosis-related myelosis. The patient age at surgery ranged from 22 to 68 years, with an average of 45.7 years. Eighteen of the patients were males and two were females. Immunostaining for MMPs was performed on frozen sections of the tissues to classify the samples based on the number of immunopositive cells. MMP levels in the resected tissues were also quantified by western blotting and densitometry. The concentrations were compared with the visual imaging of the discs. $X$ ray-based side view images revealed a high ratio of MMPs, due to an increase in osteophytes. Further, when low intensities were observed by MRI, and also when the protrusion of intervertebral discs was substantial, MMP levels were considerable. Together, these results implicate MMPs in the metamorphosis of cervical intervertebral discs, and that the metamorphosis is strongly correlated with changes in MRI and $\mathrm{X}$ ray-based side view imaging.
\end{abstract}

Key words : cervical intervertebral discs, matrix metalloproteinases (MMPs)

\section{Introduction}

The etiology of the metamorphosis and ectopia of human lumbar intervertebral discs remains unclear. Mechanical stress has been proposed as a cause, although the associated biological reactions that lead to the molecular mechanisms of this condition are unknown. The role of matrix metalloproteinases (MMPs), which destroy cartilage matrix, or a cytokine that induces the generation of MMPs, is now receiving attention ${ }^{1-4)}$. The mature intervertebral disc is originally without blood vessels. New blood vessels are created following intervertebral disc metamorphosis due to aging or the rupture of the fibrous ring. These newly created blood vessels have been implicated in the absorption process associated with hernial organizations ${ }^{5)}$. Articular cartilage undergoes destruction, matrix disassembly and repair, and synovitis, irrespective of the cause of destruction. Because intervertebral

\footnotetext{
1) Department of Orthopaedic Surgery, Showa University School of Medicine, 5-8 Hatanodai-1, Shinagawa-ku, Tokyo 142-8555, Japan.

2) Department of Oral Pathology, Showa University School of Dentistry.
} 
discs contain few cell components, they are not very susceptible to external effects, unlike synovial membranes or blood components. At the same time, this structure can undergo metamorphosis because of aging at a relatively early time. In addition, the metamorphosis of intervertebral discs is closely related to the mobile nature of the vertebrae. In particular, degenerative metamorphosis poses clinical problems in the form of various types of vertebral diseases.

In an intervertebral disc, a fibrous ring is arranged in a layered panel shape, surrounding a gel-form nucleus pulposus in the center. The upper and lower ends of the ring are closely connected in fiber shapes to cartilage end panels, which are made of vitreous cartilage. In the external layer of the fibrous ring, long-shaped, fibrous ring-bud-form cells incline toward the inside, where they become rounded, adopting the shape of cartilage cells. These cells actively synthesize proteoglycan or collagen, thereby maintaining the integrity of the extracellular matrixes. During embryogenesis, blood vessels enter from the centrum of the vertebra through the endplate of the cartilage, but in adults, ordinary blood vessels are not admitted. Nutritional supply is implemented via expansion through the endplates or fibrous rings. Much remains unknown regarding the mechanism involved in the breakdown of an intervertebral disc matrix with the described characteristics.

MMPs are enzymes that breakdown extracellular matrices. Thus far, 25 MMPs have been discovered, and based on their molecular structure and matrix specificity, they have been divided into 5 groups: collagenases, gelatinases, stromelysins, membrane-type MMPs (MT-MMP), and one other group ${ }^{4)}$. MMPs have a highly specific function in metabolism, namely the breakdown of interstitial matrix, and are involved in various physiological and pathological processes involving tissue breakdown and repair. MMPs are synthesized in epithelial cells and fibroblasts, and after being secreted as latent-type enzymes to the cell exterior, they can breakdown specific matrixes following activation. In these cases, activation is implemented by the inhibitory effect of tissue inhibitors of metalloproteinases (TIMPs). Therefore, matrix decomposition by MMPs is regulated via synthesis of MMPs, activation of MMPs, and inhibition of MMP activation by TIMPs ${ }^{6}$.

Generally, articular cartilage matrixes are broken down by protease action. Also, in the herniation of lumbar intervertebral discs, development of a hernial mass is accompanied by the production and growth of blood vessels in cartilage cells and their matrixes, as well as the infiltration of inflammatory cells, particularly macrophages. These infiltrating macrophages and cartilage cells produce MMPs within intervertebral discs that can breakdown the cartilage components. One thing that is unclear is what will be observed clinically by imaging with regard to the action of proteases in intervertebral disc destruction. Our research, therefore, sought to compare MMP analysis with visual imaging in surgical samples of cervical intervertebral discs.

\section{Materials and Methods}

\section{Patient samples}

Twenty intervertebral discs were removed from patients surgically treated for herniated cervical intervertebral discs and myelosis. The study cohort comprised 18 male patients and two female patients. Patient age at the time of surgery ranged from 22 years to 68 years, with an average of 45.7 years. There were 11 cases with herniation of the cervical intervertebral discs, 5 cases of myelosis, and 4 intervertebral dislocation/fracture cases. 
Classified according to location, there were two C3/4 cases, two $\mathrm{C} 4 / 5$ cases, $10 \mathrm{C} 5 / 6$ cases, $5 \mathrm{C} 6 / 7$ cases, and one $\mathrm{C} 7 / \mathrm{T} 1$ case.

\section{Preparations and processing of samples}

Intervertebral tissues were removed and placed in optimal cutting temperature O.C.T. compound, and snap frozen with isopentane chilled with liquid nitrogen. Frozen samples were sectioned $(4 \mu \mathrm{m})$ using a cryostat. Sections were fixed in $10 \%$ neutral formalin, and processed for hematoxylin and eosin staining (Fig. 1), azan staining, van Gieson staining, PAS staining, alcian blue staining, and mucicarmine staining, according to standard protocols.

\section{Immunohistochemistry}

Frozen sections were dried, fixed for $20 \mathrm{~min}$ in $4 \%$ paraformaldehyde-phosphate buffered saline (PBS), and washed several times with Tris-buffered saline (TBS). Endogenous peroxidase acitivity was blocked by $0.3 \%$ hydrogen peroxide in methanol at $37^{\circ} \mathrm{C}$ for 30 min. After washing with TBS, sections were incubated at $37^{\circ} \mathrm{C}$ for $1 \mathrm{~h}$ with anti-hMMP-1 $(2 \mu \mathrm{g} / \mathrm{ml})$ and anti-hMMP-3 $(2 \mu \mathrm{g} / \mathrm{ml})$ (Fuji Pharmaceutical Industry, Toyama). Following another TBS wash, sections were then incubated with biotinylated anti-mouse IgG (DAKO LSAB2 kit, USA) at $37^{\circ} \mathrm{C}$ for $10 \mathrm{~min}$. After a further wash with TBS, they were subjected to streptavidin horseradish peroxidase (DAKO LSAB2 kit, USA) for $10 \mathrm{~min}$, visualized with DAB (DAKO ENVISION kit/HRP, USA), and counterstained with hematoxylin. The scoring system was classified into 3 stages (low, mild, and high). MMP reactivity was classified as low when $25 \%$ or less of the total number of cells stained positive for MMPs; mild when 25 to $50 \%$ of cells were positive, and high when greater than $50 \%$ of the cells showed positive staining.

\section{Western blot analysis}

For Western blot analysis, 14 frozen sections were cut per sample. The sections were washed in water then placed in $0.1 \mathrm{ml}$ of protein lysis buffer $(50 \mathrm{mM}$ Tris- $\mathrm{HCl} \mathrm{pH} \mathrm{7.4,} 150$ $\mathrm{mM} \mathrm{NaCl}, 1 \mathrm{mM}$ EDTA, $1 \% \mathrm{NP}-40,0.25 \%$ Na-deoxycholate, $1 \mathrm{mM}$ phenylmethylsulfonyl fluoride: PMSF, $10 \mu \mathrm{g} / \mathrm{ml}$ aprotinin, $1 \mu \mathrm{g} / \mathrm{ml}$ leupeptin, $1 \mathrm{mM}$ sodium orthovanadate: Na3VO4, $1 \mathrm{mM}$ sodium fluoride: $\mathrm{NaF}$ ). The sections were placed on ice for $10 \mathrm{~min}$, and collected using a cell scraper. They were transferred to $1.5-\mathrm{ml}$ microcentrifuge tubes (QSP), and after $10 \mathrm{~min}$, were centrifuged at $14000 \mathrm{rpm}$ at $4{ }^{\circ} \mathrm{C}$ for $15 \mathrm{~min}$. The supernatants were recovered and total protein measurements were done using the DC protein assay (BIORAD). Denatured protein $(20 \mu \mathrm{g})$ was separated by $10 \%$ SDS-PAGE, electrotransferred onto a nitrocellulose membrane, and western blotting was performed using a standard procedure. Anti-hMMP-1/anti-hMMP-3 (Fuji Pharmaceutical Industry) was used at $2 \mu \mathrm{g} / \mathrm{ml}$. Membranes were incubated with peroxidase-linked anti-mouse IgG (Amersham, UK) and developed using ECL Western blotting detection reagents (Amersham, UK). The bands were quantified using the N.I.H image method.

\section{In situ Zymography (FIZ Method)}

Frozen sections were directly placed onto FIZ films, and incubated for $24 \mathrm{~h}$ at $37^{\circ} \mathrm{C}$ in a moist chamber. To observe the action of proteases such as MMP-2/9 on the gelatin on 
the film, the FIZ membranes were stained to visualize the areas of digestion (Fig. 2$)^{7}$.

\section{Visual Image Classification}

Simple $\mathrm{X}$ ray-based side view images and the degree of osteophyte formation

The degree of osteophyte formation was divided into 4 stages, from Grade 0 to Grade 3, based on simple $\mathrm{X}$ ray-based side view images.

Grade 0 : Cases in which no views of newly produced bones can be seen on the edge of the vertebral centrum.

Grade 1 : Cases in which views of newly produced bones, measuring $1 \mathrm{~mm}$ or less, can be seen on the edge of the vertebral centrum.

Grade 2: Cases in which views of newly produced bones, measuring $1-3 \mathrm{~mm}$, can be seen on the edge of the vertebral centrum.

Grade 3 : Cases in which views of newly produced bones, measuring greater than $3 \mathrm{~mm}$, can be seen on the edge of the vertebral centrum ${ }^{8)}$.

Simple $X$ ray-based side view images and the degree of narrowing of the intervertebral space

The degree of narrowing of inter-centrum space was divided, based on simple $X$ ray-based side view images, into 3 degrees: Grade 1 to Grade 3. For the evaluation, the average of the upper and lower inter-centrum spaces was calculated, and the height of the intercentrum space for the intervertebral discs extracted was shown in terms of the ratio to the aforementioned average. Grade 1: 100\% or more. Grade 2: From $80 \%$ to less than $100 \%$. Grade 3 : Less than $80 \%$.

\section{Degree of intervertebral disc metamorphosis in MRI}

With regard to cervical intervertebral discs, the degree of intervertebral disc metamorphosis was classified based on a brightness change grading, using MRI. Concerning the MRI evaluation method, the intensity at central portions of intervertebral discs, in T2-stressed visual images, was grouped into the following 3 grades. This grading was carried out considering that, as metamorphosis of the intervertebral discs goes on, the moisture content decreases, causing low-level brightness.

Grade 1 : Intervertebral discs that show high intensity almost evenly.

Grade 2: Intervertebral discs that partially include high intensity points but have the mixed presence of low intensity points.

Grade 3 : Intervertebral discs that indicate low intensity throughout the disk surface ${ }^{9)}$.

\section{Rear-protrusive images of intervertebral discs in MRI}

The rear-protrusive images of cervical intervertebral discs were graded by MRI, and for the purpose of morphological comparison of the rear-protrusive images of intervertebral discs in MRI, T1-stressed visual images were classified into the following 3 grades:

Grade 1: No rear protrusion is seen.

Grade 2: Although slight protrusion is observed, no images of pressing/excluding the spinal column are seen.

Grade 3 : Images of rear protrusion of intervertebral discs, and their resultant pressure application to, and exclusion of, the spinal column, can be seen ${ }^{9)}$. 


\section{Assaying Method}

Kendall's rank-correlation method was used to assay all graphs.

\section{Results}

\section{Pathological organization}

In the vertebral pulp, vitreous metamorphosis and fibrous conversion were seen, and with advanced metamorphosis, small ruptures of the vertebral pulp were seen. In some cases of advanced metamorphosis, the vertebral pulp tissues had disappeared.

Expansion and departure/opening of fibers were seen in the intermediate and internal layers of the fibrous ring, and cyst formation was also seen at this site. In advanced metamorphosis, border areas between the fiber bundles and vertebral pulp became unclear, while the dispersal of acidic mucilage polysaccharide, visualized using alcian blue, became uneven, and the polysaccharide tended to occur mainly between fibers. In addition, growth, departure/opening, and expansion of fibers were seen between the intermediate and internal layers. In cases of advanced metamorphosis, the acidic mucilage polysaccharide decreased, while fibrous conversion progressed, and vitreous metamorphosis occurred in parallel. In fibrous rings that showed significant metamorphosis, the fibrous bundle runs disappeared, while the fibrous ring matrix appeared unstructured. Many parts were positive by PAS staining.

The degree of metamorphosis in the cartilage plates was relatively small compared with that of the vertebral pulp and fibrous rings. However, where the cartilage plates were severely ruptured or partially peeled off from the edge of the intervertebral centrum, production and growth of cartilage cells were observed (Fig. 3-1.2)

In this study, the FIZ method was used to measure the localization of gelatinase activation enzymes, such as MMPs, in the tissue samples. Fig. 2 shows that the digested gelatin was principally in cartilage cells, rather than in the fibrous ring. Particularly in cases of advanced metamorphosis, more extensive areas of gelatin digestion were seen in cartilage cells. The aforementioned development indicates that gelatinase action principally occurred in cartilage cells.

\section{Comparison of MMP-positive cells by immunostaining with visual imaging}

The degree of osteophyte formation in the intervertebral discs was assessed by simple $\mathrm{X}$ ray-based side view imaging. The more osteophyte formation there was, the higher the number of MMP-immunopositive cells (Table 1). There was no significant difference between the degree of narrowing of the cervical inter-centrum space in simple $\mathrm{X}$ ray-based side view images and the ratio of MMP-positive cells (Table 2). By MRI, there was a significant difference between the degree of intervertebral disc metamorphosis and the level of MMP immunoreactivity. The lower the intensity on the MRI images, the higher the number of MMP-immunopositive cells (Table 3). A significant difference was also seen between rear-protrusive images of intervertebral discs by MRI and the MMP levels. The more advanced the herniation, the higher the number of MMP-immunopositive cells (Table 4).

Comparison of the MMP levels by western blotting with the visual imaging

A significant difference was seen between the degree of osteophyte formation and the 


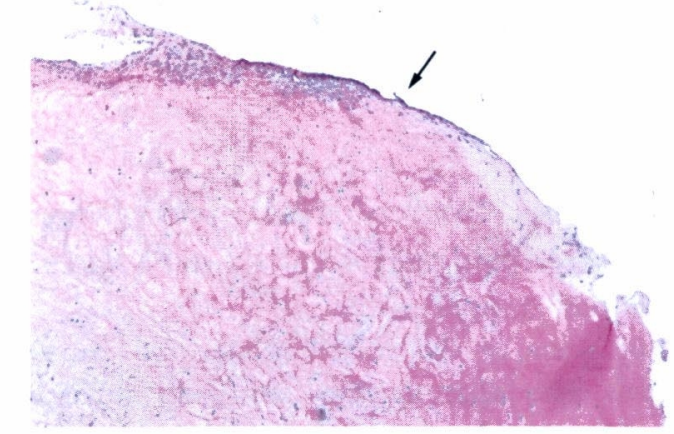

Fig. 1. H.E. staining $(\times 40)$

$\leftarrow$ cartilage cells

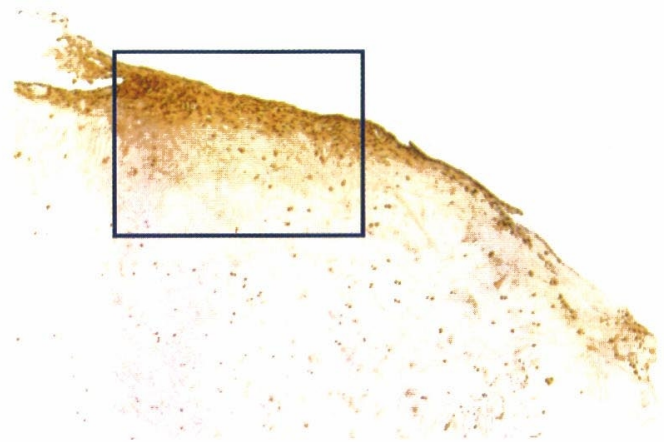

Fig. 3-1. Immunolocalization of MMP-1 $(\times 40)$

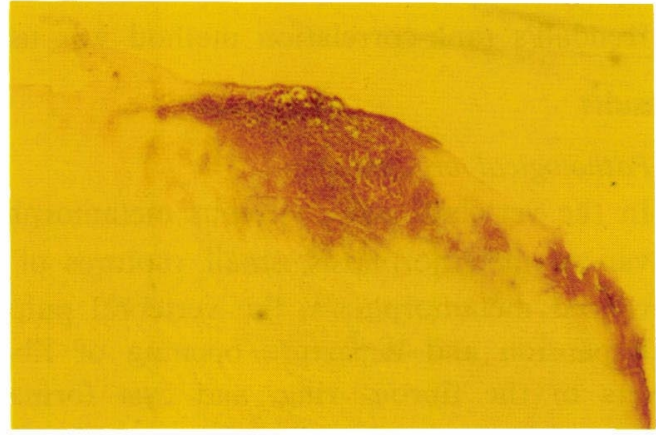

Fig. 2. MMP-FIZ method $(\times 40)$ It was further possibe to observe the localization of gelatinase activation, by staining the gelatin membrane and visualizing the digestion traces, and to facilitate gelatin reaction to protease, such as MMP-2/9.

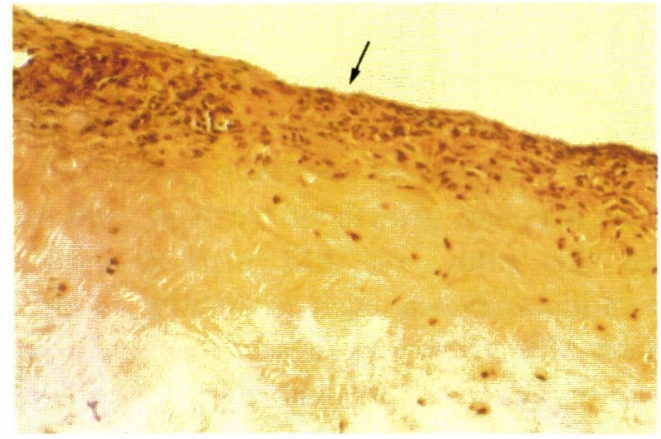

Fig. 3-2. mmunolocalization of MMP-1 $(\times 100)$

$\leftarrow$ Stained positive for MMPs in immune staining.

levels of MMPs. The more osteophyte formation there was, the higher the concentration of MMPs per tissue sample (Fig. 4-1). Although no significant difference was seen between the degree of narrowing of the cervical inter-centrum space and the MMPs in the discs, somewhat higher MMP concentrations were measured samples where there was a medium degree of narrowing of the inter-centrum space (Fig. 4-2). A significant difference was seen between the MMP concentration per sample and the degree of intervertebral disc metamorphosis. The lower the intensities by MRI, the higher the levels of MMPs by immunoblotting (Fig. 4-3). A significant difference was also seen between rear-protrusive images of intervertebral discs and concentrations of MMPs. The more advanced the herniation was, the higher the MMP levels (Fig. 4-4).

The results presented show that similar comparisons were obtained between MMP levels and visual imaging of the intervertebral discs whether the MMPs were measured by the number cells that stained positive for MMPs or by Western blotting and densitometry. 
Table 3. The degenerative degree of the disc on MRI and MMP incidence

\begin{tabular}{|c|c|c|c|}
\hline Grade & ${ }^{d}$ Low & ${ }^{d}$ Mid & ${ }^{\mathrm{d}} \mathrm{High}$ \\
\hline \multicolumn{4}{|l|}{${ }^{a}$ Grade 1} \\
\hline$(n=6)$ & $4(66.7 \%)$ & $2(33.3 \%)$ & 0 \\
\hline \multicolumn{4}{|l|}{${ }^{\mathrm{b}}$ Grade 2} \\
\hline$(n=7)$ & $1(14.3 \%)$ & $2(28.6 \%)$ & $4(57.1 \%)$ \\
\hline \multicolumn{4}{|l|}{${ }^{\mathrm{c}}$ Grade 3} \\
\hline$(n=7)$ & 0 & 0 & $7(100.0 \%)$ \\
\hline \multicolumn{4}{|l|}{$p<0.01$} \\
\hline \multicolumn{4}{|c|}{ 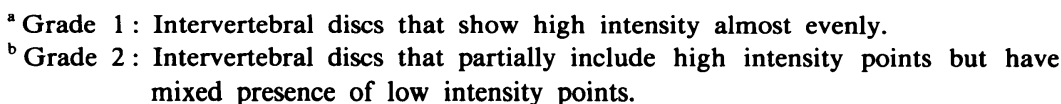 } \\
\hline
\end{tabular}

Table 4. Posterior protrusion of the disc on MRI and MMP incidence

\begin{tabular}{|c|c|c|c|}
\hline Grade & ${ }^{d}$ Low & ${ }^{\mathrm{d}} \mathrm{Mid}$ & ${ }^{d}$ High \\
\hline \multicolumn{4}{|c|}{${ }^{\mathrm{a}}$ Grade 1} \\
\hline$(n=6)$ & $5(83.3 \%)$ & $1(16.7 \%)$ & 0 \\
\hline \multicolumn{4}{|c|}{${ }^{\mathrm{b}}$ Grade 2} \\
\hline$(n=5)$ & 0 & $2(40.0 \%)$ & $3(60.0 \%)$ \\
\hline \multicolumn{4}{|c|}{${ }^{\mathrm{c}}$ Grade 3} \\
\hline$(n=9)$ & 0 & $1(11.1 \%)$ & $8(88.9 \%)$ \\
\hline \multicolumn{4}{|l|}{$p<0.01$} \\
\hline \multicolumn{2}{|c|}{ 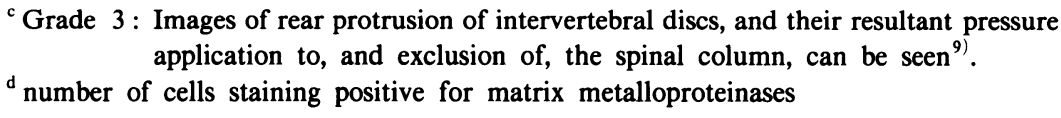 } & & \\
\hline
\end{tabular}

Osteophyte formation, which is one of the symptoms of osteoarthritis, occurs mainly in the periphery of articulations, and is coated with cartilage tissue, as revealed histologically. It has a layer of mesenchymal cells at its top surface, which presumably originates from the periosteum, and contains many blood vessels. These mesenchymal cells gradually convert to cartilage cells and expanded cartilage cells. Based on bone formation via osteoblasts, and bone absorption via osteoclasts, osteophytes are shaped due to so-called endochondral ossification, which is accompanied by active blood vessel formation. This generation of new vasculature is a key to the ossification of cartilage. In the normal ossification of cartilage mechanism, bFGF in cartilage is excluded from the cartilage due to the action of an MMP. The separated bFGF becomes a migration/multiplication element for vascular endothelial cells, creating fresh blood cells. Because of this, cartilage gradually becomes fully formed bone. In other words, the formation of osteophytes testifies to the significant involvement of an MMP in the process from cartilage formation to ossification. In this research, a significant difference was seen between the degree of osteophyte formation, revealed by 


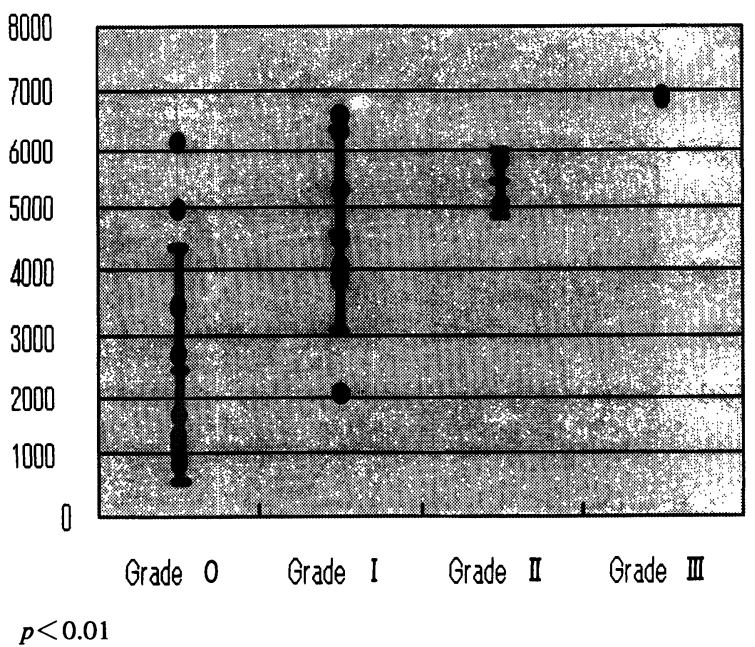

Fig. 4-1 N.I.H. image technique (X-ray) The degree of osteophytegenesis and MMPs.

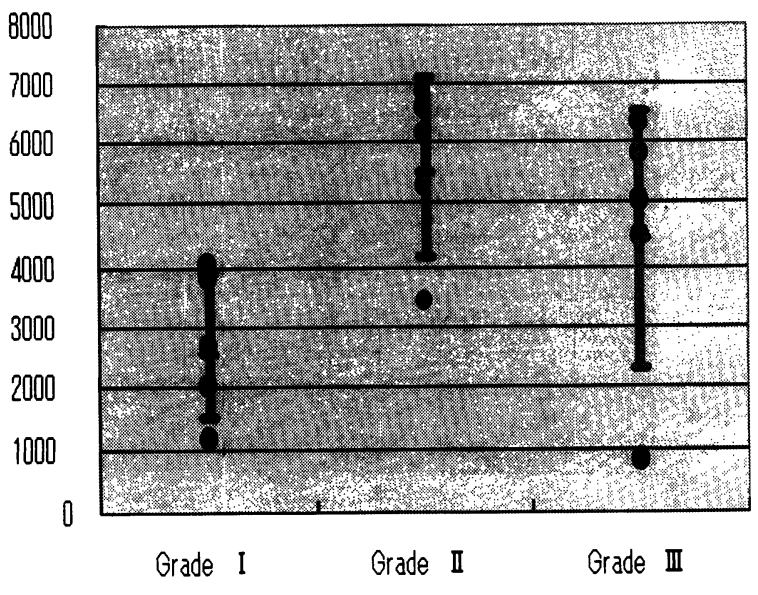
n.s.

Fig. 4-2 N.I.H. image technique (X-ray)

The degree of narrowing of corpus vertebral interstitium and MMP incidence.

clinical visual imaging, and the presence of MMPs in the intervertebral disc tissue. This finding can be likened to the involvement of MMPs in the process of osteophyte formation through endochondral ossification. In addition, considering that MMPs were found in cartilage cells rather than in the fibrous ring by immunostaining, and that staining for MMPs at the cartilage endplates was particularly intense, we propose that MMPs are involved in the formation of osteophytes through ossification from the endplates. Further, various kinds of hormones and cytokines are deeply involved in the osseocartilaginous osteophyte formation process, as they can serve as a factor promoting the generation of MMPs ${ }^{10)}$. Additionally, the transforming growth factor-b (TGF-b) can induce osteophyte formation when it is administered into joints ${ }^{10)}$. At the same time, TGF-b serves as an 


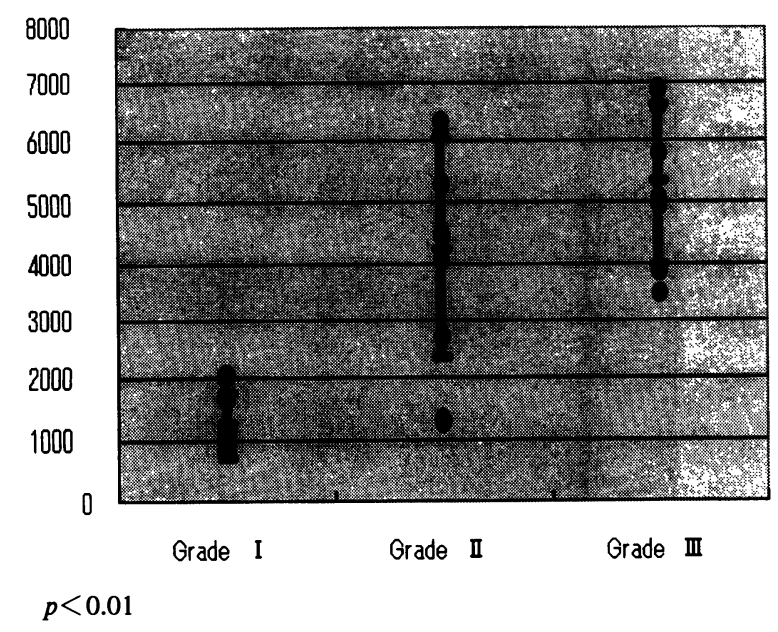

Fig. 4-3 N.I.H. image technique (MRI)

The degenerative degree of the disc and MMP incidence.

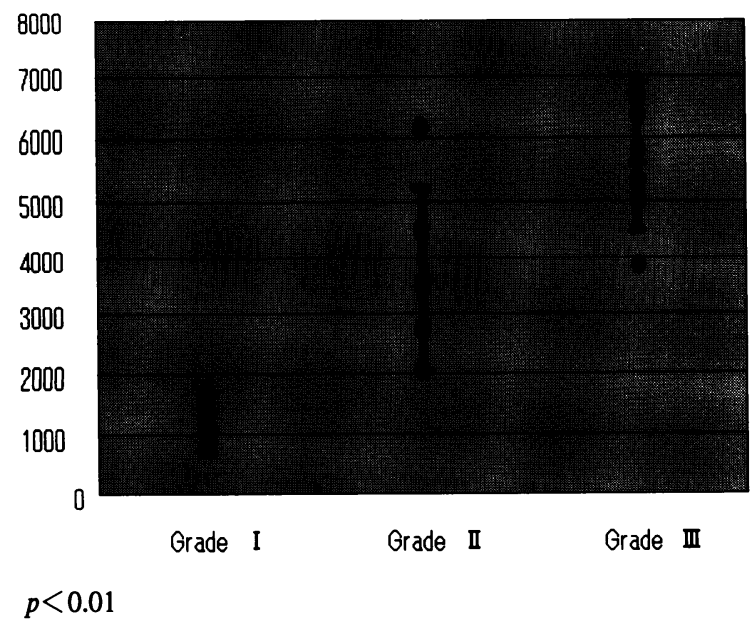

Fig. 4-4 N.I.H. image technique (MRI)

Posterior protrusion of the disc in MRI and MMP incidence.

inducing factor for MMP-2/9 and is probably involved directly or indirectly with MMPs to promote osseocartilagenous ossification.

Herniation of intervertebral discs is a disease, with the main symptoms being pain from the nerve root, perception handicaps, and motor paralysis. Cases of spontaneous natural remission are not infrequent. The recent development of MRI and other visual imaging diagnostic methods, has allowed the phenomena of herniation contraction, or its absorption over time, to be clarified, and these phenomena are considered to be the causes of natural remission. The mechanism of absorption of the herniation has been extensively studied. According to these reports, as well as to histological studies of herniated discs extracted during surgery, it is proposed that herniation is absorbed due to the growth of newly formed blood vessels and to the involvement of inflammatory cells, such as macrophages, 
inflammation-related cytokines, such as IL-1, or proteoglycan-decomposing MMPs ${ }^{11)}$. Indeed, in this study, advanced cases of herniation of cervical intervertebral discs showed newly produced blood vessels and the immunostaining for MMPs was intense in vascular endothelial cells and cartilage cells. Furthermore, visual image observation revealed a significant difference between rear-protrusive images of intervertebral discs and MMP localization and amount per tissue, suggesting that MMPs are strongly implicated in the pathological metamorphosis of intervertebral discs.

Intervertebral discs have a characteristic structure in that the vertebral pulp is located in the center, surrounded by a fibrous ring in a layered structure. The vertebral pulp cells and fibrous ring cells are engaged in de novo synthesis of proteoglycan and collagen, which together form the intervertebral disc matrix. Meanwhile, the fibrous ring principally synthesizes collagen, which prevents the loss of proteoglycan, due to its layered structure. The space between runs of collagen, which extend out in a three-dimensional net-like structure, is filled with gel-like proteoglycan and water. Proteoglycan has the function of resisting the load pressure in intervertebral discs ${ }^{12)}$. When the ratio of proteoglycan to collagen declines with aging, intervertebral discs lose flexibility, becoming unable to serve as shock absorbers, and vertebral pulp becomes fibers, eventually undergoing metamorphosis ${ }^{13)}$. However, the involvement of proteoglycan metabolism in intervertebral disc metamorphosis remains largely unknown. These discs are organizations in which there are very few cells, and a large proportion is occupied by extracellular matrix. For this reason, the destruction of extracellular matrix becomes an important point in the course of intervertebral disc metamorphosis ${ }^{14)}$. In our comparison of clinical visual imaging and MMPs, presented here, a significant difference was also noticed between the degree of metamorphosis seen by MRI and the levels of MMPs. MMPs can be divided into latent and active types, and the digestion of extracellular matrix is performed by active-type MMPs. To convert latent-type MMPs to the active type, it is necessary to remove a propeptide, which involves amongst other things the combining of $\mathrm{Zn}$ and water ${ }^{15)}$. Considering that MRI imaging intensity in this disease is dependent on the moisture content of the proteoglycan, the involvement of MMPs in the metamorphosis of intervertebral discs signifies an environment with conditions suitable for conversion of latent-type to active-type MMPs. Thus, the activation of MMPs, in this disease situation may rely on the proteoglycan to supply the necessary water. As such, a close relationship between the metamorphosis diagnosed by MRI and the MMP status of the disc tissue is suggested.

We argue then that MMPs are strongly implicated in the metamorphosis of cervical intervertebral discs, and that their location and amount in the tissue correlate firmly with changes in $\mathrm{X}$ ray-based side view images and MRI results. Further, these results can be used to investigate the correlation of TIMPs and diagnostic visual imaging in this disease, as well as the involvement of.proteoglycan in the mechanism of metamorphosis. Such information will allow clinicians to implement more accurate disease classification and corresponding clinical treatments.

\section{References}

1) Haro H, Shinomiya K, Murakami S and Spengler DM : Up-regulated expression of matrilysin and neutrophil collagenase in human herniated discs. $J$ Spinal Disord $13: 245-249$ (1999)

2) Haro H, Murakami S, Komori H, Ogawa A and Shinomiya K : Chemonucleolysis with human stromelysin-1. Spine 22 : 1098-1104 (1997) 
3) Kanemoo M, Fukuda S, Komiya Y, Katsuura A and Nishioka J : Immunohistochemical study of matrix metalloproteinase-3 and tissue inhibitor of metalloproteinase-1 in human intervertebral discs. Spine 21:1-8 (1996)

4) Sato T: Mechanisms of MMPs expression and activation. Bone 15 : 109-115 (2001)

5) Haro H, Spengler DM, Shinomiya K, Komori H and Matrisian LM: The role of MMP in herniated disc degradation. Clinical Orthopaedic Surgery 35 : 565-573 (2000)

6) Takase R, Ohshima T, Nakano H, Hirakawa T, Maki K, Shibasaki Y and Irie T: Histological and enzymological study about experimental scar tissue in rat. J Showa Dent Assoc 20 : 232-240 (2000) (in Japanese)

7) Nemori $R$ and Tachikawa $T$ : A review for in situ zimography. Tissue Cult Eng 25: 361-364 (1999) (in Japanese)

8) Gen H: A clinicopathological study of cervical intervertebral discs. J Jpn Orthop Assoc 64: 572-582 (1990) (in Japanese)

9) Maruyama Y: Histological, magnetic resonance imaging, and discographic findings on cervical disc degeneration in cadaver spines: A comparative study. J Jpn Orthop Assoc 69 : 1102-1112 (1995) (in Japanese)

10) Nishida K : Osteophyte formation and cytokine. J Joint Surg 18 : 1324-1325 (1999) (in Japanese)

11) Minamide A, Hashizume $H$, Yoshido $M$, et al: The role of cytokine in the resorption process of herniated disc. Spine Spinal Cord $14: 289-296$ (2001) (in Japanese)

12) Maroudas A: Nutrition and metabolism of the intervertebral disc, CRC Press, pp 1-37 (1988)

13) Adams $\mathbf{P}$ and Muir $\mathrm{H}:$ Qualitative changes with age of proteoglycans of human lumbar discs. Ann Rheum Dis 35 : 289-296 (1976)

14) Okuda S, Ariga $\mathrm{K}$ and Yonenobu $\mathrm{K}$ : Mechanisms of aging and development in intervertebral disc. Spine Spinal Cord $14: 258-264$ (2001) (in Japanese)

15) Takaishi H, Nemoto $O$ and Shinmei $M$ : Aging change of the cartilage. Geriatr Med $33: 409-414$ (1995) (in Japanese)

[The publication of this paper was given a priority date] 